\title{
Menschenbilder und Anthropologie in der Bioethik
}

\author{
Marcus Düwell
}

Online publiziert: 20. January 2011

(C) Die Autor(en) 2010. Dieser Artikel ist auf Springerlink.com mit Open Access verfügbar.

Zusammenfassung In der deutschen medizinethischen Debatte spielen Verweise auf Menschenbilder eine große Rolle. Dabei haben diese Verweise ganz unterschiedliche argumentative Funktionen: Bisweilen sollen mit Bezug auf ein Menschenbild direkt moralische Normen begründet werden, aber Verweise auf Menschenbilder können auch andere Funktionen haben. Problematisch erweist sich jedoch, dass der Verweis auf ein „Bild“ vom Menschen voraussetzt, dass in einem einzigen Bild alle Aspekte, die für das Verständnis des Menschen wesentlich sind, erfasst werden können und damit die Vermittlung zwischen deskriptiven und normativen Dimensionen als unproblematisch angesehen wird. Eine normative Begründungsfunktion des Menschenbildes ist jedoch aus verschiedenen moralphilosophischen Erwägungen heraus problematisch. Dagegen könnte eine Thematisierung eines umfassenden Verständnisses vom Menschen uns in der Tat helfen, metaphysische und ontologische Voraussetzungen moralischer Diskurse sichtbar und diskutierbar zu machen, die Bedingungen des menschlichen Handlungsraumes zu verstehen und das Moralische in einem weiteren Kontext praktischen Selbstverständnisses zu interpretieren. Die Mehrdimensionalität des Begriffs „Menschenbild“ lässt es fraglich erscheinen, ob der Begriff geeignet ist, medizinethische Fragen adäquat zu verstehen; zur Begründung von Handlungsnormen sind Menschenbilder eher ungeeignet.

Schlüsselwörter Anthropologie $\cdot$ Menschenbild $\cdot$ Menschliche Natur · Bioethik

\section{Image of man and bioethics}

Abstract Definition of the problem In German bioethical debates, we very often find references to the concept of "Menschenbild" (image of man/concept of man). But those references to an "image of man" can have different functions: sometimes they shall directly justify moral norms, while sometimes they refer to aspects of the human being that are

M. Düwell $(\square)$

Ethiek Instituut, Universiteit Utrecht,

Heidelberglaan 8, 3584 CS Utrecht, Niederlande

E-Mail: m.duwell@uu.nl 
only indirectly relevant for moral questions. Problematic is, however, that the concept presupposes that in one "image" all aspects of an understanding of the human being are visible at the same time and that tensions between descriptive and normative aspects of an understanding of the human being are already solved. Argumentation To justify moral norms by reference to an "image of man" is problematic from a metaethical perspective. An explication of more comprehensive understandings of the human being could, however, help us to make metaphysical and ontological assumptions in ethical debates visible and disputable. Such an explication could help us to understand the preconditions of our agency and to understand moral questions in the broader context of the practical self-understanding of human beings. Conclusion It is the central aim of this paper to raise doubts whether the multidimensional concept "Menschenbild" is appropriate to understand bioethical questions adequately. For a justification of moral norms, "Menschenbilder" are not useful.

Keywords Philosophical anthropology Concept of the human being · Human nature Image of man $\cdot$ Bioethics

\section{Einleitung}

In der deutschen medizinethischen Debatte spielt der Verweis auf Menschenbilder eine nicht unwesentliche Rolle. Die Berufung auf Menschenbilder geschieht durch ganz unterschiedliche Gruppen und in verschiedenen thematischen Zusammenhängen. So ist von einem „christlichen Menschenbild“ die Rede, es wird das „freiheitliche Menschenbild des Grundgesetzes“ gepriesen und es werden das „mechanistische Menschenbild“ der Biologie, das „ökonomistische Menschenbild des Utilitarismus“ oder das „Menschenbild der Biomedizin" kritisiert. Ob die Neurowissenschaften zu einem Wechsel des Menschenbildes Anlass geben, ist ebenfalls Gegenstand von Diskussionen. Charles Taylor kritisiert das ,atomistische Menschenbild“ [12] und manche halten dem ein „relationales Menschenbild“ entgegen; auch wird gefordert, dass die „Endlichkeit des Menschen“, die „Verletzlichkeit“ und „Abhängigkeit des Menschen“ in unserem Menschenbild angemessen berücksichtigt werden müssten. Dabei scheinen diejenigen, die sich auf Menschenbilder berufen oder diese kritisieren, anzunehmen, dass bereits deutlich sei, was man genau unter einem „Menschenbild“ $\mathrm{zu}$ verstehen habe und welche argumentative Funktion der Verweis auf ein Menschenbild haben kann. Es ist auffallend, dass die Rede vom Menschenbild zwar nicht ausschließlich ein Phänomen der Medizin- und Bioethik ist, dort aber mit so großer Selbstverständlichkeit verwendet wird wie in kaum einem anderen Bereich der Ethik, Sozialphilosophie oder Politischen Philosophie. Im Folgenden werde ich zunächst einige Funktionen der Berufung auf Menschenbilder unterscheiden, dann relevante Traditionslinien und begriffliche Fragen besprechen und schließlich eigene systematische Überlegungen präsentieren, um mit einigen Überlegungen zu den Herausforderungen der „Life Sciences“ zu schließen.

Eine kurze terminologische Vorbemerkung: Wenn ich von „Anthropologie“ rede, ist stets die philosophische Anthropologie gemeint, also der systematische Versuch, philosophisch Verbindliches über den Menschen zu sagen. Mit dem Begriff „Menschenbild“ beziehe ich mich auf die vielfältigen Verwendungsweisen, die dieser Terminus in den aktuellen Debatten hat; diese fallen keineswegs mit der Anthropologie zusammen; im nächsten Abschnitt werden einige mögliche Verständnisse unterschieden. Es soll also nicht irgendein Menschenbild verteidigt oder kritisiert, sondern es soll die Frage gestellt werden, was eigentlich genau geschieht, wenn der Begriff in ethischen Diskussionen in Anspruch genommen wird. 
Es ist das Ziel dieses Beitrags, Zweifel an der selbstverständlichen Verwendung des Begriffs „Menschenbild“ in der Medizinethik zu wecken.

\section{Einige Funktionen von Menschenbildern}

Im Folgenden sollen aus heuristischen Gründen drei mögliche normative Funktionen von Menschenbildern unterschieden werden. Es werden dabei gleichsam einige Topoi zitiert, um diese Funktionen deutlich zu machen. Dabei geht es nicht darum, eine Diskursanalyse der Verwendungsweise dieser Topoi vorzunehmen, sondern es geht darum, Möglichkeiten der Berufung auf Menschenbilder in systematischer Absicht zu skizzieren.

\section{Menschenbild als normative Begründung}

„Euthanasie ist mit dem christlichen Menschenbild (nicht) vereinbar.“ Eine solche Aussage suggeriert, dass normative Fragen um die Zulässigkeit von Euthanasie durch einen Verweis auf ein Menschenbild beantwortet werden oder zu beantworten seien. In moralischen Diskursen wäre nach diesem Verständnis das zugrunde liegende Menschenbild dafür entscheidend, wie wir über strittige normative Fragen denken oder denken sollten. Dieser Gedanke liegt auch einer Berufung auf das ,freiheitliche Menschenbild des Grundgesetzes“ zugrunde. Dabei wird angenommen, dass die Menschenwürde-Formel das Menschenbild des Grundgesetzes zum Ausdruck bringt. Die Menschenwürde wäre dann dasjenige Menschenbild des Grundgesetzes, das einen normativen Rahmen darstellt; konkrete Normen müssten dann im Lichte dieses Menschenbildes bestimmt werden. Solche Menschenbilder können etwa als normatives Leitbild im Sinnes eines Resultats geschichtlicher Erfahrung betrachtet werden. In dieser Verwendung haben Menschenbilder dann direkt die Funktion einer normativen Begründung moralisch strittiger Fragen.

\section{Menschenbild als Begründung der Moralfähigkeit des Menschen}

Ein anderer Kontext ergibt sich etwa bei Diskussionen um Neurowissenschaften, Evolutionsbiologie oder genetischen Determinismus. Verweise auf das „Menschenbild der Biologie“ oder das „Menschenbild der Neurowissenschaften“, die Kritik an biologischem oder naturalistischem Reduktionismus oder Determinismus thematisieren die Frage, inwiefern der Mensch überhaupt als moralisches Subjekt zu denken ist, inwiefern er Willensfreiheit besitzt, sein Handeln kontrollieren kann, inwiefern ihm Verantwortung zugesprochen werden kann oder inwiefern er zu moralischer Reflexion befähigt ist. Hier geht es also nicht darum, im Verweis auf ein Menschenbild Antworten auf strittige normative Fragen zu finden, sondern darum, ob sich diese normativen Fragen überhaupt stellen. Nehmen wir etwa an, es würde sich im Zuge der neurowissenschaftlichen Forschung herausstellen, dass der Mensch zu moralischer Reflexion und zur Übernahme von Verantwortung nicht in der Lage ist (wir blenden einmal die Frage aus, ob ein solcher Nachweis überhaupt denkbar ist). Ein solches deterministisches Menschenbild würde nicht zur Begründung moralisch strittiger Fragen beitragen, sondern ein solches Menschenbild würde moralische Fragen gegenstandslos machen. In dieser Verwendung sollen Verweise auf Menschenbilder Antworten auf die Frage geben, ob der Mensch überhaupt ein moralfähiges Wesen ist und sich moralische Fragen stellen kann, ohne dass damit diese Fragen bereits beantwortet würden. 
Heuristische Funktionen von Menschenbildern

Während die beiden genannten Funktionen von Menschenbildern eine deutlich rekonstruierbare argumentative Funktion für den ethischen Diskurs haben, sind zahlreiche Verweise auf Menschenbilder argumentativ uneindeutig. Man kann hier etwa an die Kritik an einem atomistischen Menschenbild von Charles Taylor denken [12], an Verweise darauf, dass das Menschenbild der Moderne die Endlichkeit des Menschen vergessen habe oder dass einige ethische Positionen ein dualistisches Menschenbild unterstellen würden. Man kann hier aber auch an die Genealogie von Foucault denken, die ja in der Bioethik durchaus beliebt ist $[1,5]$. Solche Studien versuchen, undurchschaute Vorannahmen über den Menschen in normativen Diskursen aufzuweisen. Die Diskussion um Charles Taylor hat etwa dazu geführt, dass die Sozialontologie des Liberalismus und seine möglichen historischen Wurzeln diskutiert wurden. Eine solche Form der Ideologiekritik (falls dieser altmodische Ausdruck hier verwendet werden darf) bietet selber jedoch keine Rechtfertigung einer eigenen normativen Position, ist also in normativer Perspektive unterbestimmt. Menschenbilder können also moralisch relevante oder faktisch wirksame Annahmen über den Menschen explizit machen, die normative Konsequenzen wären dann aber in einem eigenen normativethischen Diskurs auszuweisen.

\section{Menschenbilder in der Ethik: Begriffe und Traditionen}

Diese erste kurze Beschreibung von Verwendungsweisen von Menschenbildern deutet also auf sehr verschiedene argumentative Funktionen hin. Das wirft die Frage auf, was genau mit dem Verweis auf Menschenbilder erreicht werden soll. Dieser Diskurs um Menschenbilder ist nicht selbstverständlich. Der holländische Begriff „,mensbeeld“ etwa spielt in medizinethischen Diskussionen keine wesentliche Rolle. Im englischen Sprachraum ergibt sich unmittelbar die Frage der adäquaten Übersetzung: „Image of man“ ist ein künstlicher Begriff, der in ethischen Diskussionen auch gänzlich unüblich ist. Eine mögliche Übersetzung für „Menschenbild“ wäre: ,concept of the human“ oder ,concept of human nature“. Diese Übersetzung deutet aber bereits einen wesentlichen Unterschied zum Begriff „Menschenbild“ an: Der Begriff „Menschenbild“ versucht den Zugang zum Menschen in der Form eines Bildes zu erfassen. Im Bild sind alle Aspekte des Menschen synchron anwesend und können mit einem Blick erfasst werden; „,concept of the human“ meint dagegen einen „Begriff“ vom Menschen, verweist also auf ein strukturiertes und rational bereits erschlossenes Konzept.

Mit der Fokussierung auf das Bildliche wird es schwierig, die Mehrdimensionalität der Thematisierungsweisen des Menschen überhaupt zu erfassen, und noch schwieriger, die verschiedenen Hinsichten über den Menschen zu reden, methodisch transparent zu verstehen. Die wesentliche Vermittlungsleistung des „Menschenbildes“ scheint ja darin zu bestehen, dass eine normativ-deskriptive Einheit vorausgesetzt oder konstruiert wird, die in einem Bild kumuliert. Die Rede vom „Menschenbild“ scheint zu suggerieren, dass wenn man weiß, wer der Mensch ist, man auch weiß, wie man mit ihm umgehen solle. Dabei ist das Deskriptive dann nicht als (natur-)wissenschaftlich beschreibbares Faktum zu verstehen, sondern man müsste annehmen, dass unsere Wahrnehmung von der Welt bereits evaluativ strukturiert ist. Dass man dies in einem Bild fangen will, scheint auch einen Verweis auf die „Imago Dei“Tradition darzustellen, die Gottesebenbildlichkeit. Wenn der Mensch nur angemessen gesehen wird, wenn man ihn als Ebenbild Gottes denkt, dann wird durch das Bild zugleich auch die legitime Umgangsweise mit dem Menschen bestimmt. Diese deskriptiv-normative Vermittlungsleistung des Menschenbildes legt auch den Gedanken einer menschlichen Natur 
nahe, in der moralische Fragen mit Verweis auf die „Natur des Menschen“ beantwortet werden. Man könnte daher versucht sein, die Rede von Menschenbildern als Ausdruck eines anthropologischen Essentialismus zu verstehen, also als Ausdruck einer dem Menschen wesentlichen Natur. In dieser perfektionistischen Tradition würde man dann versuchen, das richtige Handeln aus einer Vorstellung besonders gelingenden menschlichen Lebens herzuleiten. Während die Rede von der menschlichen Natur versucht, auf moralische Fragen eine universalistische Antwort zu geben, und die Tradition der Gottesebenbildlichkeit versucht, einem Bild vom Menschen normative Verbindlichkeit zu verleihen, wird in der neueren Diskussion jedoch häufig von einer irreduziblen Pluralität von Menschenbildern ausgegangen. Die Frage nach dem moralisch Richtigen verschöbe sich dann auf die Frage, ob es ein richtiges oder adäquates Menschenbild gibt. Wenn aber alle normativen Aussagen von Menschenbildern abhängen und wenn zugleich von einer irreduziblen Pluralität von Menschenbildern auszugehen ist, so wären moralische Diskurse eigentlich nur denkbar als Versuche, einander zum Wechsel des Menschenbildes zu bewegen: Einigung zu moralischen Fragen wäre dann nur möglich als anthropologische Konversionserfahrung. In moralischen Diskursen ginge es immer darum, das Gesamt unseres Bildes vom Menschen zur Diskussion zu stellen. Es wäre nicht möglich, sich auf die Diskussion der Geltung eines Moralprinzips oder einzelner moralischer Normen zu beschränken.

Über den Zusammenhang von „Imago Dei“ und Menschenwürde wird nun auch die Menschenwürde-Formel zu einem Teil des Menschenbild-Diskurses. Dieser Zusammenhang macht es jedoch fraglich, ob die Rede von Menschenbildern geeignet ist, die deskriptiv-normative Vermittlung wirklich zu leisten, die sie zu leisten verspricht; die MenschenwürdeFormel versucht dies meines Erachtens nicht. Wenn wir die Menschenwürde-Tradition in dieser Perspektive einer Vermittlung zwischen dem Normativen und dem Wesen des Menschen sehen, finden wir zumindest die folgenden Modelle (vgl. [2]):

1. In verschiedenen religiösen Kontexten bezeichnet Menschenwürde den Heilsstatus des Menschen gegenüber Gott. Dieser Heilsstatus ist ethisch nur dann relevant, wenn die Erfüllung moralischer Normen als Weg zur Erreichung des Heils verstanden wird, das Moralisch-Normative also eine Funktion im religiösen Selbstverständnis des Menschen wird.

2. In der Renaissance bei Pico de la Mirandola bezeichnet die Menschenwürde den kosmologischen Status des Menschen. Pico geht es überhaupt nicht um die Frage, wie man mit Menschen umgehen muss. Es handelt sich um eine metaphysisch-deskriptive Bestimmung des Menschen.

3. Bei Kant wird die rationale Natur als Wert an sich selbst angesehen, worin die Forderung nach Respekt vor der Menschheit begründet wird. Es handelt sich um eine transzendental-notwendige Zuschreibung von Freiheit an den Menschen, mit der normative Folgerungen verbunden werden.

4. In der Universalen Menschenrechtserklärung ist Menschenwürde die normative Basis der Zuschreibung von Menschenrechten. Es wird eine Wertzuschreibung an den Menschen vorgenommen, aus der normative Konsequenzen gezogen werden.

Diese kurze Übersicht zeigt, dass auch in der Würdezuschreibung nicht ohne Weiteres eine Einsicht in das Wesen des Menschen mit Forderungen nach moralisch legitimen Umgangsweisen mit dem Menschen verbunden sind, die gewissermaßen ,,in einem Blick“ zu erfassen sind, wie es der Begriff des Menschenbildes suggeriert. Wir müssen vielmehr die Vermittlung noch als eine Aufgabe ansehen, die eigens zu leisten wäre. 


\section{Zum Verhältnis von Ethik und Menschenbild}

Eine der Schwierigkeiten des Begriffs „Menschenbild“ liegt also darin, dass der Begriff suggeriert, eine normativ-deskriptive Vermittlung sei bereits geleistet. Problematisch daran ist, dass hier sowohl die Rekonstruktion verschiedener Perspektiven einer Thematisierung des Menschlichen als auch der begriffliche Status von Menschenbildern schwer transparent bestimmt werden können. Begrifflich gehören zu unserem Bild vom Menschen die vielfältigsten Formen von Überzeugungen und Annahmen: Wir haben Überzeugungen über den „Sinn menschlichen Lebens“, über „Formen gelingenden Lebens“, über moralische „Verpflichtungen, die Menschen gegenüber Menschen haben“, Annahmen über wesentliche Fähigkeiten des Menschen und vieles mehr. Von einem Menschenbild erwartet man, dass es eine Art „comprehensive doctrine“ vom Menschen sei [10]. Nun wird anscheinend angenommen, dass es selbstverständlich sei, dass moralische Fragen nur im Horizont solcher umfassenden Verständnisse des Menschlichen beantwortet werden. So wurde etwa in der Hirntod-Debatte häufig angenommen, es sei völlig unstrittig, dass die wesentliche Frage darin bestehe, wann der Mensch tot sei. Ebenso wurde unproblematisch unterstellt, dass es beim Umgang mit Embryonen um die Frage ginge, wann der Mensch ein Mensch sei. Zunächst geht es in beiden Diskussionskontexten jedoch um die normativ-ethische Frage, wie man Menschen in verschiedenen Lebensphasen behandeln soll und welche Verpflichtungen wir gegenüber Menschen in den jeweiligen Lebensphasen haben. Es ist keineswegs selbstverständlich, dass diese normativ-ethische Frage durch den Verweis auf ein Menschenbild beantwortet werden kann, das den Menschen in all seinen anthropologischen Dimensionen erfasst. Die anthropologische Kritik an bestimmten Standardauffassungen in der Moralphilosophie, die man etwa bei Theda Rehbock findet, führt geradezu zu einer Transformation von Ethik in Anthropologie [11].

Dabei fragt sich zunächst, worauf sich eine solche normativ-anthropologische Bestimmung des Menschen genau bezieht: Es könnte darum gehen, eine gelungene Form menschlichen Lebens im Sinne einer perfektionistischen Ethik normativ auszuzeichnen. Eine solche Form anthropologischer Ethik wäre zumindest prima facie mit dem Respekt vor einer Pluralität von Lebensentwürfen nur schwerlich vereinbar, wie ihn das menschenrechtliche Würdekonzept fordert. Oder aber es ginge um die Bestimmung moralisch relevanter Fähigkeiten. Die letztere Konzeption würde sich auf eine normative Anthropologie in dem Sinne beziehen, dass jene Fähigkeiten des Menschen bestimmt werden, die für ihn als praktisches Wesen unverzichtbar sind. Der moralische Schutz wäre als Schutz der Realisierungsmöglichkeiten dieser Fähigkeiten zu denken, womit eine prinzipiell liberale Pluralität von Realisierungsmöglichkeiten vereinbar ist. Martha Nussbaum hat in ihren Arbeiten aus den 1990er Jahren in diesem Sinne den Schutz menschlicher Fähigkeiten auf einer minimalen Anthropologie aufgebaut, einer Theorie der menschlichen Natur [7]. In den letzten Jahren hat sie diese Theorie menschlicher Natur stillschweigend durch die Theorie des „overlapping consensus“ im Sinne von Rawls ersetzt [8]. Dieser Verweis auf wesentliche menschliche Fähigkeiten begründet aber weder die Frage, welche Fähigkeiten moralisch relevant sind, noch, warum sie moralisch schützenswert sind. Die Herausarbeitung der anthropologischen Bedeutsamkeit bestimmter Fähigkeiten begründet noch nicht, dass es eine allgemeine moralische Verpflichtung gibt, die Realisierung dieser Fähigkeiten moralisch zu schützen. Selbst wenn man annimmt, dass der moralische Schutz sich darauf bezieht, relevante menschliche Fähigkeiten zu schützen, so ist damit noch nicht gesagt, dass die Bestimmung dieser Fähigkeiten durch eine Anthropologie im Sinne einer umfassenden Bestimmung des Menschen erfolgen müsse, vielmehr ist es ebenfalls möglich, den moralischen Schutz auf spezifisch 
moralisch relevante Fähigkeiten zu beziehen, deren Ausweis dann eine spezifischere Frage der Moralphilosophie wäre.

Diese Überlegungen wollen also grundsätzlich Zweifel daran wecken, ob man in einem Verweis auf ein Menschenbild und auf die Anthropologie eine direkte Begründung der Berechtigung moralischer Verpflichtungen erzielen kann. Doch es gibt eine Reihe von anderen Dimensionen, in denen eine Diskussion von Menschenbildern durchaus relevant ist:

1. Kritik am anthropologischen Bias von ethischen Theorien. Moralisch informativ wäre der Nachweis, dass bestimmte ethische Forderungen oder Prinzipien von problematischen Annahmen über den Menschen abhängig sind. Falls eine solche Abhängigkeit gezeigt werden könnte, so wäre sie für die jeweilige ethische Theorie ein seriöses Problem. Dabei denke ich aber nicht an Platitüden nach dem Muster, dass der Utilitarismus den Menschen auf Ökonomismus oder Nutzenmaximierung reduziert (das ist nämlich nicht der Fall). Ebenso wenig reduziert Kant den Menschen auf seine Vernunft. Und selbst wenn Kant oder der Utilitarismus derart reduktionistisch zu Werke gehen würden, wäre dies ethisch nur dann interessant, wenn man zeigen könnte, dass diese Annahmen für diese Theorien notwendig und zugleich problematisch sind. Deutlich interessanter sind dagegen Versuche zu zeigen, inwiefern bestimmte ontologische oder metaphysische Annahmen oder Zeit- und Geschichtsvorstellungen notwendige Voraussetzung sind, um zentrale moralische Begriffe wie Würde oder Verantwortung kompetent verwenden zu können. Hier gibt es in der Tat mögliche Kandidaten von Annahmen über den Menschen, die überhaupt ein Potential haben, ethische Theorien ernsthaft in ihrem Geltungsanspruch zu kritisieren.

2. Das Verständnis des Handlungsraums des Menschen. Für jede ethische Diskussion über legitime Handlungsforderungen ist es informativ zu wissen, wo die Grenzen der menschlichen Handlungsmöglichkeiten liegen. Neben empirischen Kenntnissen geht es dabei auch um grundlegende Bestimmungen von Voraussetzungen im Menschen als Handlungssubjekt und um Begrenzungen von Veränderungsmöglichkeiten des Menschen. Ein Verständnis dieser Grenzen und Abhängigkeiten des Menschen sagt uns aber noch nicht, was er tun soll und wie wir mit dem Menschen umgehen sollen.

3. Die Anthropologie als Vermittlung der verschiedenen Dimensionen der Thematisierung des Menschen [13]. Die Anthropologie, auch in ihrer klassischen Gestalt bei Plessner oder Gehlen, wollte die Vermittlung verstehen zwischen Einsichten der Biologie und der Naturwissenschaften über die natürliche Basis des Menschen und deren Naturgeschichte mit den verschiedenen kulturellen Äußerungen des Menschen und der praktischen Dimension menschlicher Existenz inklusive der moralischen Dimension [4, 6, 9]. Gemäß eines solchen Verständnisses wäre die Aufgabe der Anthropologie als Lehre vom Menschen in einem umfassenden Sinne grundsätzlich von der Ethik als Lehre vom richtigen Handeln zu unterscheiden. Anthropologie geht es dann um ein umfassendes Verständnis des Zusammenhangs aller Dimensionen. Ob ein solches Verständnis möglich ist und an welche Voraussetzungen es gebunden ist, will ich dahingestellt lassen. Es zeigt aber, dass der systematische Ort der Anthropologie ganz anders zu bestimmen wäre als der einer ethischen Rahmentheorie.

Ich habe damit kurz einige Perspektiven aufgezeigt, in denen anthropologische Grundlagenreflexionen ethisch durchaus bedeutsam werden könnten. Diese Perspektiven ergeben sich nur, wenn man darauf verzichtet, Ethik als angewandte Anthropologie verstehen zu wollen, oder in einer Lehre von dem, was den Menschen ausmache, bereits die normative Frage nach dem richtigen Umgang mit dem Menschen beantwortet sieht. Die Frage danach, was der Mensch sei, ist laut Kant die Grundfrage der Philosophie. Sie zielt auf die Vermitt- 
lung aller Dimensionen. Die normative Ethik zielt nicht auf das Ganze der menschlichen Existenz, sondern nur auf die Frage, wie wir handeln sollen und welche Verpflichtungen wir gegenüber einander haben. Wenn wir das wissen, dann wissen wir noch nicht alles, was man über den Menschen wissen kann. Gerade diese umfassende Frage nach dem Menschen wäre aber überhaupt als eine komplexe Aufgabe der reflexiven Vermittlung aller möglichen Thematisierungen des Menschen zu begreifen. Der Begriff des Menschenbildes suggeriert, dass diese umfassende Vermittlung im Verständnis des Menschen bereits vorhanden wäre und nicht mehr eine Aufgabe für den Menschen darstellt. Die Berufung auf Menschenbilder in normativer Absicht könnte insofern geradezu als eine Beleidigung für den Menschen aufgefasst werden, insofern sie den Mangel an Bereitschaft ausdrückt, diese Besonderheit des Menschen wirklich ernst zu nehmen. Während heutige Theologen und Juristen, aber auch viele andere glauben, dass ihnen ein christliches oder andersartiges Menschenbild zu direkter Nutzung in normativer Orientierung zur Verfügung steht, wusste die Tradition, dass der Mensch ein Geheimnis ist, und kannte die jüdische Tradition selbst das Verbot sich ein Bildnis von ihm zu machen.

\section{Die Herausforderungen der Life Sciences}

Im Sinne eines Ausblicks sei auf eine zentrale Dimension der Life Sciences im Hinblick auf unser Verständnis vom Menschen verwiesen. ${ }^{1}$ Die Rede vom Menschen hat stets eine Doppelstruktur: Als „menschlich“ bezeichnen wir Mitglieder einer biologischen Gattung, aber wir benutzen das Adjektiv auch als einen Wertbegriff. Wenn Kant von der „Menschheit in Deiner Person" redet, denkt er nicht an eine biologische Kategorisierung, sondern meint mit „Menschheit“ einen komplexen Wertbegriff. Wenn wir also in moralischer und auch rechtlicher Perspektive vom Menschen reden, dann verweisen wir mit „Mensch“ auf ein Wesen, dem Würde und Rechte zukommen. Traditionell wurde nicht angenommen, dass die Zuordnung dieses Wertbegriffs auf bestimmte biologische Erscheinungsformen nennenswerte Schwierigkeiten bereitet, zumindest hat Kant es nicht für nötig empfunden, eine Richtlinie zu formulieren, wie man bei der Anwendung des Begriffs der „Menschheit“ in der empirischen Welt vorzugehen habe. In Bezug auf die Chimären hingegen ist genau dies ein zentrales Problem. Die biologische Gattungsbestimmung führt direkt bestimmte normative Konsequenzen mit sich; besonders deutlich wird dies daran, dass alle Rechtsvorschriften einen Unterschied zwischen rechtlichen Regelungen für Tiere und Menschen machen, tertium non datur.

Eine der wesentlichen Herausforderungen der Life Sciences besteht nun darin, dass die Zuordnung von einem Wertbegriff des Menschen zur biologischen Erscheinung nicht mehr unproblematisch in der lebensweltlichen Erfahrung vermittelt ist. Vielmehr muss diese Vermittlung begrifflich geleistet werden und verdankt sich dann einem bewussten Reflexionsakt. Solange moralische und rechtliche Regelungen diese Herausforderung nicht als solche verstanden haben, sind systematische Missverständnisse mit weitreichenden Konsequenzen unvermeidlich. Auch hier ist die ganzheitliche Rede vom „Menschenbild“ äußerst hinderlich um das Problem als solches überhaupt zu verstehen.

\footnotetext{
${ }^{1}$ Diese Überlegungen gehen zurück auf das Projekt CHIMBRIDS, einem europäischen Projekt unter Leitung von Jochen Taupitz über ethische Fragen in Bezug auf Chimäre und Hybride [3].
} 
Open Access Dieser Artikel unterliegt den Bedingungen der Creative Commons Attribution Noncommercial License. Dadurch sind die nichtkommerzielle Nutzung, Verteilung und Reproduktion erlaubt, sofern der/die Originalautor/en und die Quelle angegeben sind.

\section{Literatur}

1. Brenner A (2006) Bioethik und Biophänomen. Den Leib zur Sprache zu bringen. Königshausen und Neumann, Würzburg

2. Düwell M (2010) Menschenwürde als Grundlage der Menschenrechte. Z Menschenr 1:64-79

3. Fiester A, Düwell M (2009) Ethics. In: Taupitz J, Weschka M (Hrsg) CHIMBRIDS - Chimeras and hybrids in comparative European and international research: scientific, ethical, philosophical and legal aspects. Springer, Berlin, S61-79

4. Gehlen A (1986) Der Mensch. Seine Natur und seine Stellung in der Welt. Aula, Wiesbaden

5. Gehring P (2006) Was ist Biomacht? Vom zweifelhaften Mehrwert des Lebens. Campus, Frankfurt a. M.

6. Lindemann G (2009) Das Soziale von seinen Grenzen her denken. Velbrück, Weilerswist

7. Nussbaum MC (1992) Human functioning and social justice: in defence of Aristotelian essentialism. Polit Theory 20:202-246

8. Nussbaum MC (2006) Frontiers of justice. Disability, nationality, species membership. Belknap Press, Cambridge

9. Plessner H (1975) Die Stufen des Organischen und der Mensch. Einleitung in die philosophische Anthropologie. Suhrkamp, Frankfurt a. M.

10. Rawls J (1998) Politischer Liberalismus. Suhrkamp, Frankfurt a. M.

11. Rehbock T (2005) Personsein in Grenzsituationen. Zur Kritik der Ethik medizinischen Handelns. Mentis, Paderborn

12. Taylor C (1992) Negative Freiheit? Zur Kritik des neuzeitlichen Individualimus. Suhrkamp, Frankfurt a.M.

13. Wimmer R (1995) Anthropologische Ethik. Erkundungen in unübersichtlichem Gelände. In: Demmerling C, Gabriel G, Rentsch T (Hrsg) Vernunft und Lebenspraxis. Philosophische Studien zu den Bedingungen einer rationalen Kultur. Für Friedrich Kambartel. Suhrkamp, Frankfurt a. M., S 215-245 